Moore provides a basic introduction to crater morphology, lunar chronology, mascons, transient lunar phenomena, regolith formation and the recent space exploration programmes. I would have liked to have seen one or two coloured geological maps (a typical example could have been the US Geological Survey map of the Copernicus quadrangle) and maybe a detailed contour map would have been fun (NASA's lunar topographic orthophotomap of the Apollo 15 Hadley region would do). Additional detail about surface temperature variation, transient atmospheres and the present meteorite influx rate would also not have been amiss.

We all have our favourite crater and I was surprised that mine, Giordano Bruno (which might have been formed as recently as July 18,1178 ), doesn't even rate a mention. However Moore has produced an ideal book for the amateur observer, and anyone who ever fancies turning a telescope or a pair of binoculars onto the Moon will find this atlas an excellent starting point and guide.

Despite the grandiose title given to the series by Mitchell Beazley I think the two atlases, especially The Moon, fall well into the amateur division of the astronomy book league. I must say, though, that at $£ 6.95$ each (in the US \$14.95, Rand McNally) their value for money will be hard to beat.

David $W$. Hughes is a Lecturer in Physics and Astronomy at The University of Sheffield.

\section{SCIENTIFIC BOOKSHOP}

H.K. LEWIS can supply works in all branches of Pure and Applied Science. Catalogues on request. Please state interests.

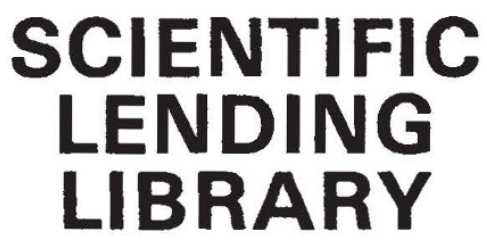

Annual Subscription from $\mathbf{8 8 . 5 0}$ (Available in U.K. only)

Reduced rates for multiple subscriptions.

Prospectus post free on request.

Quarterly List of New Books and new editions added to the Library sent post free to subscribers regularly.

\section{H.K. LEWIS \& Co. Ltd. 136 GOWER STREET, LONDON, WC1E 6BS}

\title{
Guidance for the observant amateur
}

\section{Patrick Moore}

A Complete Manual of Amateur Astronomy. By P. Clay Sherrod. Pp. 319. ISBN hbk 0-13-162115-7; ISBN pbk 0-13-162107-6. (Prentice-Hall: 1981.) Hbk $\$ 24.95$, £18.70; pbk \$10.95, £8.20. The Practical Astronomer. By Colin A. Ronan. Pp. 153. ISBN UK 0-333-31245-7; ISBN US 0-02-604500-1 . (Macmillan, London/Macmillan, New York: 1981.) £8.95, \$20.

At.THOUGH no book can be really regarded as complete, Clay Sherrod's Manual is certainly exhaustive, and it contains much up-to-date information which cannot be found elsewhere in convenient form. The author is a well-known amateur astronomer, and his experience will be found to be very useful to others.

Topics covered range from telescope selection and maintenance to naked-eye work (meteor observation, for example); studies of the Moon and planets; photoelectric photometry, and all kinds of astronomical photography. The sections dealing with equipment are of special value. Many amateurs tend to believe that setting up a photoelectric photometer is too much of an undertaking; reading this book may cause a change of outlook. The author stresses, rightly so, that the modern amateur has to be much more specialized than his predecessor of half a century ago.

There are, unfortunately, some blemishes, and the weakest parts of the book are those dealing with the Moon and planets. In the lunar section, no mention is made of transient lunar phenomena, even though it is here that the amateur can carry out his most valuable work. In some ways the information given is out of date; for instance, it is no longer believed that lunar rills are "dried beds of water flow" - this idea was abandoned many years ago, and analyses of the lunar samples brought home by the Apollo astronauts and the Russian Luna probes have shown that there are no hydrated materials on the Moon. The map of Mars given on p.141 is, frankly, of no use, and the list of Martian features includes many canals and other markings which do not exist at all - so that the amateur will be hard pressed to locate them! The most surprising statement is that in Saturn's system, "the satellites Titan, Mimas and Hyperion are those that you should study most during transit events". Any observer using a modest telescope to watch a transit of Mimas or Hyperion would indeed have to have keen vision.

There are comparable mistakes in the stellar lists. On p.204, it is said that Antares varies from magnitude 0.9 to $1.8-$ in fact its fluctuations are too slight to be detected without using a photometer or some such device; Alpha Cassiopeiae never rises to magnitude 1.4 , and even its variability is questionable (Kukarkin's catalogue omits it); presumably $n$ Aquilae should be $\eta$ Aquilae, $\mathrm{R}$ Geminorum should be $\mathrm{U}$ Geminorum, and so on. These errors are trivial in themselves, but in a book aimed at providing a guide for amateurs they can be highly misleading.

All in all, it seems that the proof-reading has been inadequate, and that the author is not fully conversant with some of the latest research. But the merits of the book far outweigh the defects, and there is nothing which cannot be put right in the next edition. When this has been done, the book may well become a standard work.

Colin Ronan's book, The Practical Astronomer, is much more elementary, and aimed at the younger reader. As well as providing a general introduction to astronomy, it contains many practical demonstrations and experiments; the text is clear and riveting, and the illustrations excellent. A book to be strongly recommended.

Patrick Moore is an author and astronomer. His recent books include The Moon, Jupiter (with Garry Hunt) and The Atlas of the Universe, all published by Mitchell Beazley.

\section{Cosmic methadone}

\section{William H. Press}

The Edge of Infinity: Naked Singularities and the Destruction of Spacetime. By Paul Davies. Pp.194. ISBN UK 0-460-04490-7; ISBN US 0-671-44063-2. (Dent/Simon \& Schuster: 1981.) $£ 7.95, \$ 14.50$.

"BEYOND black holes to the end of the universe", trumpets the cover blurb. The author, with more modest affect, relates a conversation among colleagues bemused at the plethora of black-hole books: " 'Whatever next?' someone asked. 'The naked singularity!' came a prompt reply. So I have written, with some amusement, a book about naked singularities".

A naked singularity, in modern relativity theory, is a region of spacetime where gravitational forces become infinite, crushing matter to infinite density, but from which light signals can escape, rendering the singularity visible. The singularity inside a black hole is not "naked" the choice of this word is usually attributed to Roger Penrose - but is, rather, modestly clothed behind an opaque "event horizon" (the surface of the black hole) through which outward light signals cannot propagate. The big-bang singularity from which our Universe expanded is, however, naked: the $3 \mathrm{~K}$ cosmic microwave background is a signal from that singularity (or, at least, from as close to that event as the opacity of dense, ionized matter will allow us to look). 